\title{
Structural Characterization of Carane Derivative Stereoisomers - Potent Local Anesthetics
}

\author{
J. Grochowski ${ }^{a}$, P. Serda ${ }^{a}$, M. Pasenkiewicz-Gierula ${ }^{b}$, \\ R. CZARneCKI ${ }^{c}$, T. LIBrowski ${ }^{c}, \mathrm{~S}$. LOChYŃSKI $^{d}$ \\ AND B. FRACCKOWIAK ${ }^{d}$ \\ ${ }^{a}$ Regional Laboratory, Jagiellonian University \\ Ingardena 3, 30-060 Kraków, Poland \\ ${ }^{b}$ Institute of Molecular Biology, Jagiellonian University, Kraków, Poland \\ ${ }^{c}$ Department of Pharmacodynamics, CM JU, Kraków, Poland \\ ${ }^{d}$ Institute of Organic Chemistry, Biochemistry and Biotechnology \\ Wrocław University of Technology, Wrocław, Poland
}

\begin{abstract}
The paper reports on structural investigation and phase analysis of a newly synthesized potent local anesthetic with chiral molecular structure. Absolute structure and absolute configuration on four chiral centres was determined using microcrystalline single-crystal diffraction with anomalous scattering of X-ray radiation azimuthal scan technique. Phase analysis for new compound (KP23SS) and its epimer (KP23RS) was carried out using classical and synchrotron radiation powder diffraction. Enantiopurity of the bulk material was verified for both isomers by comparison of experimental and simulated high-resolution powder diffraction diagrams. The presence of two new polymorphic phases of KP23RS was documented. Comparative conformational analysis was carried out using differential Fourier synthesis and least-squares molecule overlap technique. A model of epimeric disorder was discussed for the homochiral phase.
\end{abstract}

PACS numbers: 82.30.Qt, 61.10.-i 


\section{Introduction}

Chiral pharmaceuticals are built of molecules possessing one or more chiral centres. Since 1956, when Pfeiffer documented his thesis: "lower efficient dose of drug implies larger stereospecific difference in its enantiomer action" [1], chiral molecules have been in focus of pharmacologists [2], pharmaceutical and chemical industry [3], and legislative agencies working on standardization of medical products [4-6]. Production growth rate of chiral pharmaceuticals reaches now more than $10 \%$ per year, especially with employment of asymmetric catalysis [7] and, recently, genetic engineering [8]. During the last decade, the development of chiral drugs is the result of effort of multidisciplinary teams [9]. Research area covers physiological activity of isomers, their interaction and influence of chirality on administration of transdermal drugs (see for example [10-12]). International rules [4-6] require sophisticated procedures for proving stability and purity of stereoisomers in vitro and in vivo. Multi-directional interest in chiral drugs results in incompatible nomenclature. Therefore some definitions are included in the paper.

A chiral centre is created by an atom with substituents or ligands not superimposable on its mirror image, it has no symmetry operations of the second kind, i.e., mirror plane, centre of inversion, roto-inversion axis.

- Absolute configuration - the spatial arrangement of the atoms of a physically identified chiral molecular entity (or group) and its stereochemical description - chirality sense.

- The chirality sense denoted R(ectus) or S(inister) defines the absolute configuration of stereoisomer. Absolute configuration is assigned by X-ray anomalous scattering or circular dichroism (CD) experiment.

- Conformation - spatial arrangement (shape) of a molecule of given configuration defined by the set of its torsional angles.

- Stereoisomers with inverted all chiral centres are called enantiomers. Inversion of one of multiple chiral centres gives epimers. Epimers form a subset of diastereoisomers.

- Diastereomers are isomers with multiple chiral centres that differ in configuration at one or some, but not all, chiral centres.

- Racemate is a 1:1 mixture of two enantiomers.

At present, about $70 \%$ of local anesthetics are chiral. Chiral resolution, i.e., separation of enantiomerically pure forms, of several racemic anesthetics resulted in lower toxicity of the enantiopure form [13].

The investigated compound, expressing strong local anesthetic activity (-)-3-[2-hydroxy-3-( $N$-isopropylamino)-propoxyimino]-cis-carane hydrochloride $\left(\mathrm{C}_{16} \mathrm{H}_{30} \mathrm{~N}_{2} \mathrm{O}_{2} \cdot \mathrm{HCl}\right)$, acronym $\mathrm{KP} 23$ [14-17], is synthesized partly from components of Pinus sylvestris turpentine [18]. Both parts of the molecule: carane head 
and isopropyl side chain, may appear in stereoisomeric forms. Figure 1 shows connectivity and atom numbering scheme within the molecule. The presence of three chiral centres at carbons C13, C14, and C16 allows, theoretically, for the existence of 8 stereoisomeric forms (each centre may appear in $\mathrm{S}$ or $\mathrm{R}$ absolute configuration). The isopropyl side-chain possesses one chiral centre, at carbon C6, implying the possibility of 2 stereoisomers.

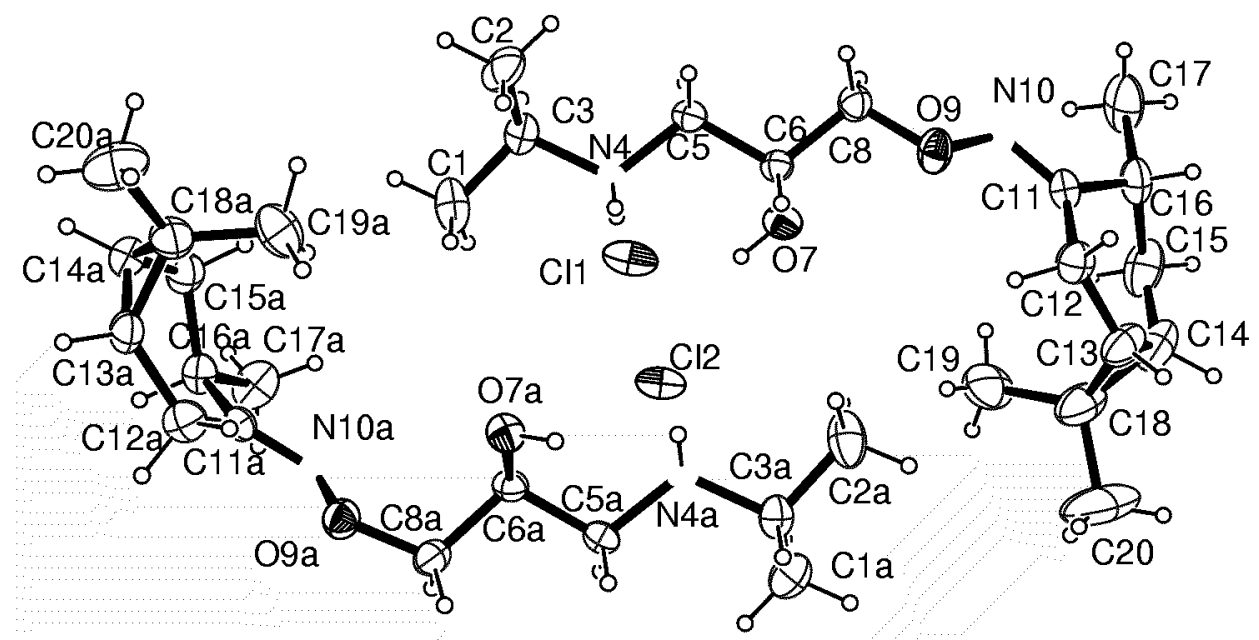

Fig. 1. Epimeric form of (-)-3-[2-hydroxy-3-( $N$-isopropylamino)-propoxyimino]-cis-carane hydrochloride $\left(\mathrm{C}_{16} \mathrm{H}_{30} \mathrm{~N}_{2} \mathrm{O}_{2} \cdot \mathrm{HCl}\right)$ with the atoms numbering scheme and thermal vibration ellipsoids. The picture was made with ORTEP-3 program.

Crystal structure of KP23RS [14] revealed that this compound crystallizes in triclinic system (space group $P 1$ ), with two epimers in the unit cell. In both molecules, the absolute configuration at carane carbons is identical $(\mathrm{C} 13-\mathrm{R}$, $\mathrm{C} 14-\mathrm{S}, \mathrm{C} 16-\mathrm{R})$, whereas at $\mathrm{C} 6$ atom of the side-chain it is opposite $(\mathrm{R}, \mathrm{S})$ in both molecules of the epimer. Independent two-way stereospecific syntheses [19] were carried out to obtain enantiopure isomers. The resulting compounds were homochiral (denoted KP23SS and KP23RR) with identical chemical formula $\mathrm{C}_{16} \mathrm{H}_{30} \mathrm{O}_{2} \mathrm{~N}_{2} \cdot \mathrm{HCl}$.

\section{Single-crystal X-ray structure analysis of KP23SS}

KP23SS crystallizes from a mixture of hexane and acetone (both anhydrous) giving small, colourless platelets with typical grain size below $0.05 \mathrm{~mm}$. The crystals are plastic and most of them are twins or overgrown - manifesting by non-uniform mosaicity. Attempts at separating grains resulted in smearing out rather than cleavage. The sample selected for single crystal diffraction experiment 
was an irregular plate $0.03 \mathrm{~mm}$ thick and $0.15 \times 0.09 \mathrm{~mm}$ wide. Diffraction data were collected using CAD-4 (Enraf-Nonius) 4-circle diffractometer and $\mathrm{Cu} K_{\alpha}$ graphite monochromatised radiation. In order to improve the counting statistics the maximum measuring time for a single reflection was extended to 180 seconds, which was possible due to good radiation stability of the sample. A total of 4130 independent reflections were collected with 3800 for $I / \sigma(I)>2$. Two intensity standards collected every hour were used to correct intensity fluctuations of the data. The data reduction was carried out using XCAD4 program from the WinGX crystallographic package [20]. Data were corrected for Lorentz polarization and semi-empirical absorption correction was calculated by the program XABS. The structure was solved by direct methods using SHELXS program in space group $P 1$ (lattice parameters $a=7.4024(2) \AA, b=7.5552(2) \AA, c=17.2493(6) \AA$, $\left.\alpha=83.26(3)^{\circ}, \beta=85.56(2)^{\circ}, \gamma=85.66(2)^{\circ}\right)$. It is noticeable that the parameters have close values to already determined unit cell parameters of KP23RS (Table). Similarly to KP23RS, the asymmetric unit consists of two independent molecules forming a dimer. Intermolecular hydrogen bonds involving nitrogen, chlorine and oxygen atoms bind neighbouring molecules into dimers. A perspective drawing of the dimer with thermal vibration ellipsoids is given in Fig. 2.

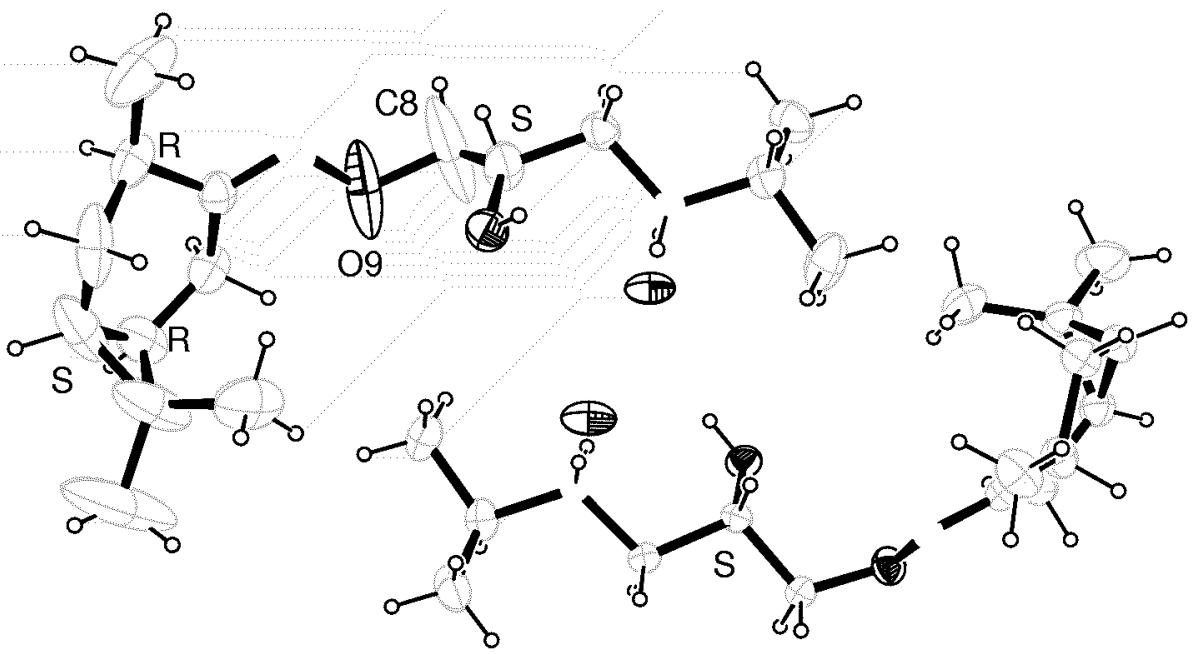

Fig. 2. Homochiral structure of KP23SS. Absolute configuration at chiral centres in the side-chains is identical $(\mathrm{S})$ in both molecules.

The absolute structure was determined using anomalous scattering of $\mathrm{Cu}$ radiation on chlorine atoms. Using the structural model, pairs of reflections with the largest Bijvoet difference $I(h)-I(-h)$, resulting from anomalous scattering contribution to the structure factor, were selected and carefully remeasured using azimuthal scan technique [4]. The absolute configuration at three chiral centres 
TABLE

Unit cell parameters for KP23RS, its polymorph and epimer.

\begin{tabular}{|c|c|c|c|c|c|c|}
\hline$a[\AA]$ & $b[\AA]$ & $c[\AA]$ & $\alpha\left[^{\circ}\right]$ & $\beta\left[^{\circ}\right]$ & $\gamma\left[^{\circ}\right]$ & $V\left[\AA^{3}\right]$ \\
\hline \multicolumn{7}{|c|}{ KP23RS, unit cell parameters measured for various single crystal samples } \\
\hline 7.3504 & 7.5304 & 17.4922 & 82.66 & 83.82 & 84.39 & 951.20 \\
\hline 7.3600 & 7.5320 & 17.5090 & 82.62 & 83.85 & 84.48 & 953.68 \\
\hline 7.3637 & 7.5405 & -17.4865 & 82.76 & 83.91 & 84.52 & 954.44 \\
\hline 7.3680 & 7.5490 & 17.4760 & 82.76 & 84.12 & 84.64 & 956.00 \\
\hline 7.3647 & 7.5431 & 17.5020 & 82.74 & 83.88 & 84.53 & 955.70 \\
\hline 7.3600 & 7.5392 & 17.4967 & 82.69 & 83.85 & 84.42 & 954.00 \\
\hline \multicolumn{7}{|c|}{ Superstructural polymorphic form $\left(a^{\prime}=a, b^{\prime}=5 b, c^{\prime}=2 c\right)$} \\
\hline $7.352(1)$ & $37.65(1)$ & $34.94(2)$ & $82.70(3)$ & $83.98(2)$ & $84.42(1)$ & $9508(5)$ \\
\hline \multicolumn{7}{|c|}{ KP23SS unit cell parameters } \\
\hline $7.4024(5)$ & $7.5552(5)$ & $17.2493(8)$ & $83.26(5)$ & $85.56(5)$ & $85.66(5)$ & $\begin{array}{c}954.0 \\
\text { (single } \\
\text { crystal) }\end{array}$ \\
\hline
\end{tabular}

of carane heads was identical for both molecules and the same as in KP23RS. The absolute configuration on chiral centres in the side chain is $\mathrm{S}$ for both centres $\mathrm{C} 6$ and $\mathrm{C} 6 \mathrm{~A}$, as expected by synthesis. Since a stereospecific reaction rarely results in enantiomeric excess above $95 \%$, careful analysis of electron density in the vicinity of $\mathrm{C} 6$ and $\mathrm{C} 6 \mathrm{~A}$ atoms was carried out using difference Fourier synthesis. No residual density above $0.28 \mathrm{e} / \AA^{3}$ was observed on the difference Fourier map, which confirms the homochirality of the dimer. To compare the conformations of two molecules forming the dimer, root-mean-square (RMS) displacement for two least-squares (LSQ) superimposed molecules was calculated, giving the value 0.71 . This value seems rather high, because analogous parameter calculated for epimeric molecules (with opposite absolute configurations on $\mathrm{C} 6$ ) from the crystal structure of KP23RS is 0.77 . This leads to a conclusion that there are two significantly different conformations in the unit cell. Figure 3 shows superimposed carane moieties of the molecules and diverging side chains due to different torsional angles around bond $\mathrm{C} 8-\mathrm{O} 9-83.4$ and 72.0 degrees, respectively.

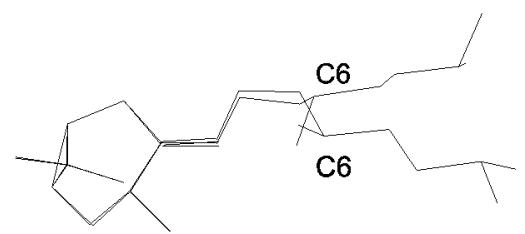

Fig. 3. Molecules of homochiral KP23SS isomer with superimposed carane moieties. 
It was observed that in one molecule, the valence angles around the chiral centre $\mathrm{C} 6 \mathrm{~A}$ have values very close to those corresponding to tetrahedral $\left(s p^{3}\right)$ hybridization, as expected, whereas atoms C5, O7, C8 together with C6 chiral centre have nearly flat configuration - the sum of the three corresponding valence bonds equals $359.7^{\circ}$. It is noticeable that C8 and O9 atoms have elongated thermal ellipsoids. Detailed analysis including the refinement of partial occupancy factors was carried out for atoms with large anisotropy of thermal vibrations: C8, O9. For the $\mathrm{C} 8$ atom bonded to $\mathrm{C} 6$ and $\mathrm{O} 9$, a disorder model was refined resulting in two alternative positions of this atom with nearly equal occupancies. The flat configuration around C6 suggests the occurrence of epimeric disorder. Large thermal vibrations observed for terminal methyl groups of carane, C17, C19, C20, could be attributed to their mobility. Disorder of the side chain is transmitted to the carane head. Statistical character of thermal displacements for all carane atoms is confirmed by the fact that RMS displacement for two LSQ superimposed carane moieties of the dimer has a low value of 0.09 .

\section{Comparison of KP23RS and KP23SS molecular structures}

Both compounds preserve a similar volume (packing density), in spite of slight variability of lattice constants. Table compares unit-cell parameters for both compounds and illustrates the variability of the unit-cell parameters of the epimeric form. The crystal and molecular structures of both compounds (KP23RS and KP23SS) reveal a significant similarity. The existence of polymorphic form and structural similarity of both compounds, as well as appearance of KP23RS form during recrystallization of enantiomerically pure KP23SS, raises three questions:

- whether a substitution of epimeric molecules is possible during the nucleation stage of crystallization;

- whether a conglomerate crystallization could occur;

- how representative the investigated single crystal sample is for the bulk material.

To answer these questions phase analysis was carried out.

\section{Powder diffraction experiments}

Larger quantities of KP23RS, KP23SS and KP23RR bulk materials were obtained by crystallization of the reaction product in the mixture of anhydrous hexane and acetone. Figure 4a illustrates environmental scanning electron microscope picture of a typical crystallite of KP23RS bulk material, with fairly well developed grains in the shape of parallelepipeds; Fig. 4b presents a spherolytic conglomerate, characteristic of KP23SS lamellae. The morphology of KP23RR is not uniform: microcrystalline spherolites and rectangular forms are visible (Fig. 4c), suggesting the presence of two different phases. 

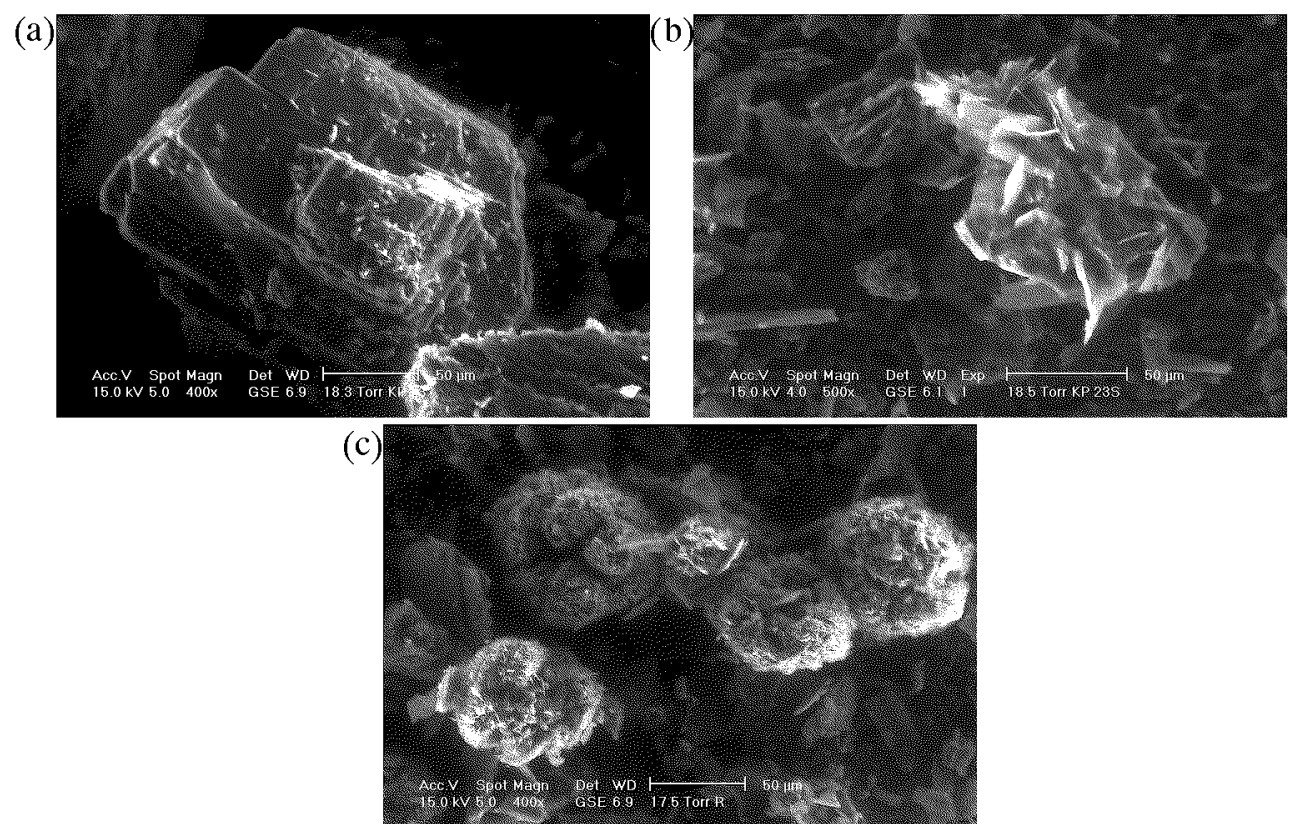

Fig. 4. SEM pictures of (a) typical crystallite of KP23RS bulk material, (b) spherolytic conglomerate, characteristic of KP23SS lamellae, (c) microcrystalline spherolites and rectangular forms of two different phases of KP23RR.

Diffraction pattern recorded with a laboratory X-ray source (Philips X'Pert, $\mathrm{Cu} K_{\alpha}$ radiation, Bragg-Brentano geometry) for KP23RS (Fig. 5a) was successfully indexed using TREOR program yielding cell parameters $(a=7.371$, $b=7.560, c=17.529 \AA, \alpha=82.63, \beta=83.85, \gamma=84.34^{\circ}$ ) with figure of merit M20 equal to 40 and F20 equal to 86 . However, the number of resolved diffraction intensity profiles within the sample diffraction limit range was too small taking into consideration the number of structural parameters of the simplest structural model (168).

A diffraction experiment for KP23RS carried out at ESRF Grenoble (BM-16 beamline, wavelength $0.803107(1) \AA$, capillary $0.8 \mathrm{~mm}$ ) resulted in a well-resolved powder pattern (Fig. $5 \mathrm{~b}$ ). The pattern was indexed using the program TMO giving the lattice parameters $a=7.361, b=7.535, c=17.514 \AA, \alpha=82.68$, $\beta=83.86, \gamma=84.34^{\circ}, \mathrm{V}=954.35 \AA^{3}$, with figure of merit M20 equal to 84.5. The pattern was consistent with that calculated from single-crystal structure. Taking into consideration the spread of lattice constants determined for different single-crystal grains (Table), the simulated pattern was calculated assuming the correctness of lattice parameters from powder pattern indexing. Due to high resolution of the SR pattern, it was possible to identify 11 peaks not belonging to the predominant structure of KP23RS; they were indexed separately giving orthorhombic parameters $(a=11.035, b=12.108, c=14.268 \AA, \alpha=90.0$, 
$\beta=90.0, \gamma=90.00^{\circ}, V=1906.5 \AA^{3}$ ). The unit cell volume of this admixture is almost exactly twice that of RS phase, which suggests the presence of yet another polymorph in the bulk material.
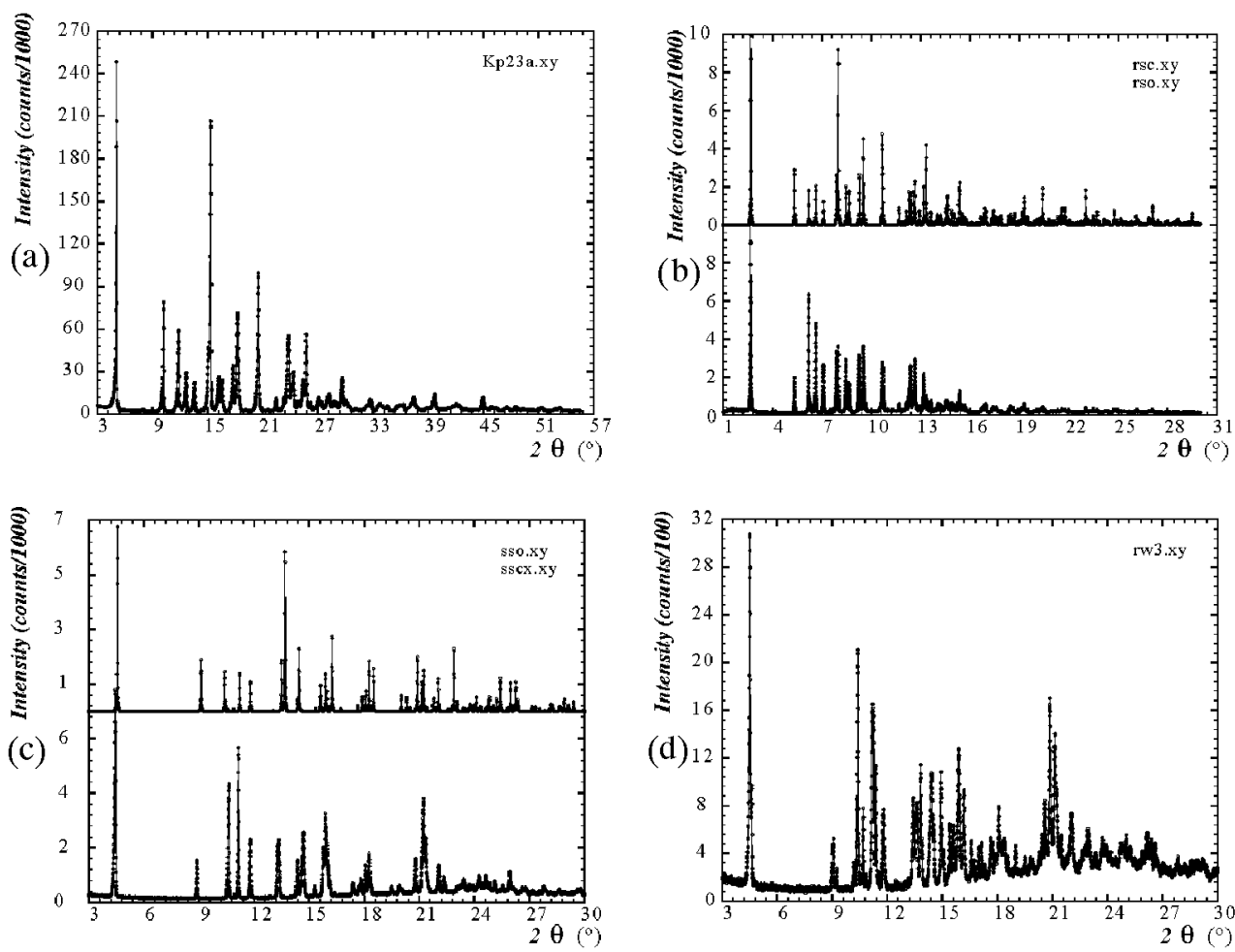

Fig. 5. Powder diffraction patterns of KP23 stereoisomers: (a) KP23RS - Philips X'Pert, Cu $K_{\alpha}$, Bragg-Brentano geometry, (b) KP23RS - synchrotron radiation, ESRF Grenoble, BM-16 beamline, wavelength $0.803107 \AA$, (c) KP23SS - synchrotron radiation, DESY-HASYLAB, B2 beamline, wavelength $1.3572 \AA$, (d) KP23RR phase mixture — synchrotron radiation, DESY-HASYLAB, B2 beamline, wavelength $1.3572 \AA$.

The measurements for KP23SS were performed at the beamline B2 at the Hamburger Synchrotron Strahlungslabor (HASYLAB), Germany [21]. A divergence reducing, cylindrical, Pt-coated mirror and a Ge(111) double crystal monochromator were used for beam conditioning. The selected wavelength was $1.3572(1) \AA$. The sample was filled in a capillary with $0.9 \mathrm{~mm}$ diameter. Intensity data were detected with a $\mathrm{NaI}$ scintillation counter with a $\mathrm{Ge}(111)$ channel cut analysing crystal mounted at the detector arm to reduce background and increase resolution.

The diffraction pattern of the KP23SS form (Fig. 5c) was successfully indexed using TREOR program yielding cell parameters $(a=7.409, b=7.396, c=$ $17.745 \AA, \alpha=81.76, \beta=85.17, \gamma=87.10^{\circ}$ ) with figure of merit M20 equal to 79 
and F20 equal to 241. The calculated pattern based on single-crystal diffraction of a microcrystalline grain matched fairly well with the experimental one, proving phase homogeneity of the species.

Two diffraction patterns of the KP23RR material (two batches synthesized by different methods) recorded under the same conditions revealed a more complex spectrum. At low Bragg angles, the peaks were apparently split (Fig. 5d), and the intensity ratio in each pair was reversed in the other batch. This indicated that each sample consists of two phases. Both phases were successfully indexed with TREOR yielding cell parameters:

phase 1: $a=5.370, b=10.360, c=17.132 \AA, \alpha=90.0, \beta=90.0$, $\gamma=90.00^{\circ}, V=953.06 \AA^{3}$,

phase $2: a=19.190, b=8.635, c=8.716 \AA, \alpha=90.0, \beta=94.66$, $\gamma=90.00^{\circ}, V=1439.49 \AA^{3}$.

After the recrystallization process, selected grains of a size suitable for single-crystal diffraction turned out to be the KP23RS variety. Additionally, single crystals of KP23RR synthesized without hydrochloride, $\mathrm{C}_{16} \mathrm{H}_{30} \mathrm{~N}_{2} \mathrm{O}_{2}$, were also examined on a KappaCCD (Nonius) diffractometer indicating turbostatic structure.

\section{Conclusions}

The epimeric form of the investigated (-)-3-[2-hydroxy-3-( $N$-isopropylamino)-propoxyimino]-cis-carane hydrochloride $\left(\mathrm{C}_{16} \mathrm{H}_{30} \mathrm{~N}_{2} \mathrm{O}_{2} \cdot \mathrm{HCl}\right)$, KP23RS, turned out to be the easiest to crystallize. Apart from the predominant triclinic phase, two polymorphic forms were detected. The observed spread of unit cell parameters is probably originating from the natural substrate (carane). A structural similarity of forms KP23RS and KP23SS facilitates the conglomerate crystallization. Conformational analysis of KP23SS structure revealed epimeric disorder around the C6 atom. For low-symmetry, non-perfect organic crystals, microcrystalline and high-resolution powder diffraction techniques with synchrotron radiation appeared to be suitable tools for structure determination and phase characterization.

\section{Acknowledgments}

The work was supported by the State Committee for Scientific Research, grant No. 4PO5F01916, and DESY-HASYLAB, category I experiment I-00-024 and by IHP - contract HPRI-CT-1999-00040 of the European Commission. The authors wish to thank Dr. A. Fitch of ESRF for test HRPD measurements and Dr. Marek Faryna of the Regional Laboratory, Jagiellonian University, for conducting experiments in Environmental Scanning Electron Microscope.

\section{References}

[1] C.C. Pfeiffer, Science 124, 29 (1956).

[2] B. Testa, M. Reist, P.A. Carrupt, Ann. Pharm. Fr. 58, 239 (2000).

[3] S.C. Stinson, Chem. Eng. News 79, 79 (2001).

[4] Test Procedures and Acceptance Criteria for New Drug Substances and New Drug Products, Food and Drug Administration: DA, Federal Register, Vol. 62, No. 227 (1997) 
[5] Test Procedures and Acceptance Criteria for New Drug Substances and New Drug Products: Chemical Substances. The European Agency for the Evaluation of Medicinal Products, MEA/CPMP/ICH/367/96 corr., 1999.

[6] Investigations of Chiral Active Substances, EMEA/CVMP/128/95.FINAL, 1998.

[7] P. De Koning, Chem. Eng. News 79, 251 (2001).

[8] T.K. Nevanen, L. Soederholm, K. Kukkonen, T. Suortti, T. Teerinen, M. Linder, H. Soederlund, T.T. Teeri, J. Chromatogr. A 925, 89 (2001).

[9] R.C. Williams, C.M. Riley, K.W. Sigvardson, J. Fortunak, P. Ma, E.C. Nicolas, S.E. Unger, D.F. Krahn, S.L. Brenner, J. Pharm. Biomed. Anal. 17, 917 (1988).

[10] E. Recanska, F. Gregan, Pharmazie 54, 68 (1999).

[11] T. Eriksson, S. Bioerkman, P. Hoeglund, Eur. J. Clin. Pharmacol. 57, 365 (2001).

[12] I.K. Reddy, T.R. Kommuru, A.A. Zaghloul, M.A. Khan, Crit. Rev. Ther. Drug Carrier Syst. 17, 285 (2000).

[13] G.A. McLeod, D. Burke, Anaesthesia 56, 331 (2001).

[14] R. Czarnecki, K. Czerwinska, K. Grochowska, J. Grochowski, T. Librowski, P. Serda, Arzneimittel Forschung 42, 1279 (1992).

[15] T. Librowski, R. Czarnecki, M. Pasenkiewicz-Gierula, J. Grochowski, P. Serda, S. Lochyński, B. Frąckowiak, Eur. J. Pharm. Sci. 11, Suppl., 113 (2000).

[16] J. Grochowski, P. Serda, M. Pasenkiewicz-Gierula, P. Talik, R. Czarnecki, T. Librowski, S. Lochyński, in: 19th European Crystallographic Meeting, Nancy 2000, Abstracts, p. 345.

[17] T. Librowski, R. Czarnecki, S. Lochyński, B. Frąckowiak, M. Pasenkiewicz-Gierula, J. Grochowski, P. Serda, Polish Journal of Pharmacology 53, 535 (2001).

[18] S. Lochyński, B. Frąckowiak, T. Librowski, R. Czarnecki, J. Grochowski, M. Pasenkiewicz-Gierula, in: 220nd IUPAC Int. Symp. on the Chemistry of Natural Products, Sao Carlos (Brazil) 2000, Abstr., p. PSA-04.

[19] B. Frąckowiak, S. Lochyński, T. Librowski, R. Czarnecki, J. Grochowski, M. Pasenkiewicz-Gierula, in: Proc. 9th Meeting on Stereochemistry, Prague 2000, p. P-64.

[20] The following computer programs were used for calculations and figures: WinGX - A Windows Program for Crystal Structure Analysis - L.J. Farrugia (1998), University of Glasgow, Scotland; SHELXL97 - Program for Crystal Structure Refinement, G.M. Sheldrick (1997), University of Goettingen, Germany; SHELXS97 - Program for Crystal Structure Solution, G.M. Sheldrick (1997), University of Goettingen, Germany; ORTEP-3 for Windows, L.J. Farrugia (1997), J. Appl. Crystallogr. 30, 565 (1997); WinPLOTR - A Graphic Tool for Powder Diffraction, T. Roisnel, J. Rodriguez-Carvajal (2001), Laboratoire Léon Brillouin, Saclay, France; TREOR90 - Program for Powder Pattern Indexing, P.-E. Werner; TMO Program for Powder Pattern Indexing, F. Kohlbeck, Technical University, Vienna.

[21] J. Grochowski, P. Serda, M. Pasenkiewicz-Gierula, R. Czarnecki, T. Librowski, S. Lochyński, B. Frąckowiak, C. Baehtz, M. Knapp, HASYLAB Jahresbericht, 2001. 